\title{
L'ALGÈBRE ARABE DANS LES TEXTES HÉBRAÏQUES (II). DANS L'ITALIE DES XV ET XVI SIÈCLES, SOURCES ARABES ET SOURCES VERNACULAIRES 1
}

\author{
TONY LÉVY
}

Nous savons encore assez mal ce que les grandes œuvres algébriques de la Renaissance italienne (Del Ferro, Cardan, Tartaglia) doivent aux traditions arabes avérées. De Fibonacci, au début du XIII ${ }^{\mathrm{e}}$ siècle, à Luca Pacioli à la fin du $\mathrm{XV}^{\mathrm{e}}$, qu'a-t-on su, qu'a-t-on exploité, des nouveaux territoires théoriques balisés par al-Khwārizmī, Abū Kāmil, al-Karajī, al-Samaw'al, al-Khayyām, Sharaf al-Dīn al-Ṭ̂̄sī pour ne citer que les noms emblématiques? Les travaux publiés sur l'abondant corpus des "traités d'abaque" italiens ne permettent pas encore de répondre clairement à ces questions. ${ }^{2}$

${ }^{1}$ Dans un précédent article, nous avons présenté un tableau des tout premiers textes algébriques composés en hébreu, entre les XII ${ }^{\mathrm{e}}$ et XIV siècles: "L'algèbre arabe dans les textes hébraïques (I). Un ouvrage inédit d'Isaac ben Salomon al-Ahdab (XIV siècle)", Arabic Sciences and Philosophy, 13 (2003): 269-301. Nous avons exposé plusieurs résultats de notre recherche dans le cadre du séminaire "Histoire de l'algèbre", organisé par le Centre d'histoire des sciences et des philosophies arabes et médiévales (CNRS), à Villejuif, au cours des années 2003-2005.

${ }^{2}$ On mettra à part le cas de Fibonacci, dont on commence à mieux évaluer le mode d'appropriation des sources arabes qui fut le sien. Voir A. Allard, "L'influence des mathématiques arabes dans l'Occident médiéval", dans R. Rashed (éd.), Histoire des sciences arabes, vol. II (Paris, 1997), pp. 199-229 (voir pp. 220-9); R. Rashed, "Fibonacci et les mathématiques arabes", Micrologus II (1994): 145-60; id., "Fibonacci et le prolongement latin des mathématiques arabes", Bolletino di Storia delle Scienze Matematiche, XXIII (2003): 55-73.

Pour une vue d'ensemble de la production algébrique italienne, on consultera W. Van Egmond, Practical Mathematics in the Italian Renaissance: A Catalog of Italian Abbacus Manuscripts and Printed Books to 1600 (Florence, 1980); R. Franci \& L. Toti Rigatelli, "Towards a history of algebra from Leonardo of Pisa to Luca Pacioli”, Janus, 72 (1985): 17-82. S'il est assuré que les écrits algébriques arabes ont nourri cette abondante production, nous sommes encore loin de voir clair dans l'histoire de cette rencontre.

Prenons un exemple significatif: le florentin Antonio de' Mazzinghi (1353-1383) fut, semble-t-il, un des plus talentueux algébristes de son temps. Telle qu'elle peut 
Les témoignages que nous avons rassemblés ici constituent un indice, et un produit, de la circulation des traditions arabes dans l'Italie de la Renaissance. Les conditions spécifiques qui ont présidé à l'apparition de ces écrits hébraïques ne doivent pas être négligées: soulignons les mouvements de populations juives (ou simplement de familles, voire d'individus) d'Espagne vers l'Italie dès la fin du XIV ${ }^{\mathrm{e}}$ siècle, qui retiennent particulièrement l'attention pour l'histoire que nous voulons reconstituer.

Deux figures émergent du tableau des écrits algébriques identifiés à ce jour; en présentant ces deux savants et leurs contributions à l'histoire de l'algèbre, nous tenterons de préciser les conditions qui ont favorisé leur intérêt pour cette discipline mathématique dans l'Italie du $\mathrm{XV}^{\mathrm{e}}$ siècle. Le premier d'entre eux, Simon Moțoț, n'a, apparemment, consulté que des sources italiennes; toutefois, il semble être à l'origine de l'intérêt que portera à l'algèbre Mordekhay Finzi; et nous devons à ce dernier le texte hébreu de l'Algèbre d'Abū Kāmil, ainsi que la traduction d'un important traité d'algèbre italien incluant des équations de degré supérieur à 2 .

\section{SIMON B. MOÏSE B. SIMON MOṬOṬ ET LE CALCUL DE L'ALĠ̈BRE}

Nous ne savons rien de la biographie de cet auteur, si ce n'est une mention indirecte de l'époque où il adresse son livre d'algèbre à Mordekhay Finzi et un autre ami: après $1446 .^{3}$ Le nom de Simon Moṭoṭ est associé à un autre texte mathématique, consacré à la propriété asymptotique de l'hyperbole. Nous avons montré que cet écrit se rattachait à une tradition arabe remontant à Sharaf al-Dīn al-Ṭūsī (XII ${ }^{\mathrm{e}}$ s. $)$, sans être en mesure, toutefois, de préciser la nature de la transmission de

\footnotetext{
être reconstituée, l'œuvre de ce maestro d'abaco comporte d'importants développements algébriques qu'on peut rapprocher des définitions figurant dans le Fakhrī d'al-Karajī; voir R. Franci, "Antonio de' Mazzinghi: An algebraist of the 14th century", Historia Mathematica, 5 (1988): 240-9, à la p. 243, et F. Woepcke, Extrait du Fakhrî. Traité d'algèbre par Abou Bekr Mohammed ben Alhaçan Alkarkhî (Paris, 1853), p. 53. Pour autant, nous ne savons rien, dans l'état actuel de la recherche, des liens qui pourraient rattacher les idées du savant arabe du $\mathrm{X}^{\mathrm{e}}$ siècle à celles de l'algébriste florentin du $\mathrm{XIV}^{\mathrm{e}}$ siècle.

${ }^{3}$ G. Sacerdote, "Le livre de l'algèbre et le problème des asymptotes de Simon Moțoț”, Revue des études juives, 27 (1892): 91-105, 28 (1893): 228-46, 29 (1894): 111-26; voir vol. 27, p. 93.
} 
l'arabe à l'hébreu. ${ }^{4}$ Un autre indice témoignant des contacts - sans doute indirects - de ce Simon Motot avec la culture scientifique arabe concerne sa bibliothèque familiale: dans un manuscrit médical arabe du XII ${ }^{\mathrm{e}}$ siècle, transcrit en caractères arabes, apparaît une marque de possession rédigée en hébreu par un certain Simon b. Moïse Moṭot, lequel indique que le manuscrit faisait partie d'un héritage familial. ${ }^{5}$ On peut raisonnablement identifier ce personnage avec l'auteur du livre d'algèbre (le nom de Moṭoṭ est assez rare); pour autant, rien ne nous permet d'affirmer que Simon Moțoṭ pouvait lire des livres arabes, ni même qu'il connaissait des rudiments d'arabe.

Quoi qu'il en soit, le livre du Calcul de l'algèbre (Heshbon ha-Alzibra) ne comporte pas la moindre référence explicite à la science arabe ou à un auteur arabe. ${ }^{6}$ Il n'y est question que "des livres des chrétiens".

Présentons le contenu de l'ouvrage, avant de commenter ce que nous savons des conditions de sa composition. Les lignes introductives offrent d'intéressantes remarques sur les fondements de l'algèbre, tels que l'auteur les a compris:

Après la louange à Dieu [. . .], je commence et je dis: il convient que tu saches que les chrétiens, dans le calcul de l'algèbre, considèrent certaine partie d'un problème dont le nombre n'est pas connu, et ils en font, dans leur calcul, une chose pleine et entière (davar ehad shalem), qu'ils appellent cosa (q.w.s.'). Par ce mot, ils veulent signifier deux aspects: une chose pleine et entière et [en même temps] quelque chose de caché que nous ne connaissons pas. C'est pourquoi, moi aussi, je ferai de même

${ }^{4}$ T. Lévy, "L'étude des sections coniques dans la tradition médiévale hébraïque. Ses relations avec les traditions arabe et latine", Revue d'histoire des sciences, 42 (1989): 193-239; voir pp. 197-209.

${ }^{5}$ P. SJ. Van Koningsveld, “Andalusian-Arabic manuscripts from Christian Spain: A comparative intercultural approach", Israel Oriental Studies, 12 (1992): 75-110, voir p. 103, n 94. P. Van Koningsveld suggère que Simon Moțoṭ pourrait être un descendant de Samuel b. Sa'adya Moțoț, un exégète connu, commentateur d'Ibn Ezra, qui a vécu à Guadalajara vers 1370, et qui a traduit de l'arabe en hébreu le Livre de la foi sublime d'Abraham ibn Daoud. Pour séduisante qu'elle soit, cette filiation ne repose, pour l'heure, que sur le rapprochement entre les deux noms.

${ }^{6}$ Il y a plus d'un siècle, G. Sacerdote en a donné une traduction française (Sacerdote, "Le livre de l'algèbre") à partir du texte hébreu de Berlin, Staatsbibliothek Preussischer Kulturbesitz, MS Hebr. 244, fols. 113a-120b. On connaît quatre autres copies: Mantoue, Biblioteca Comunale, MS Ebr. 10, fols. 122b-133a; Florence, Biblioteca Mediceo-Laurenziana, MS Plut. 88. 46, fols. 46a53b; Parme, Biblioteca Palatina, MS De Rossi 205, fols. 117a-119b; Amsterdam, Portugees Israelietisch Seminarium Ets Haim, MS HS.EH. 47D20, fols. 221a-225a. Nous citons, pour notre part, le texte du manuscrit de Mantoue, en le confrontant, si nécessaire, aux leçons proposées par les autres copies. 
dans ma traduction que voici et je le désignerai par le nom de 'chose' (davar). Le produit d'une chose par elle-même, ils l'appellent censo (s.i.n.s.w). J'ai interrogé leurs grammairiens sur le sens de ce mot; ils m’ont dit qu'il désigne un nombre déterminé, entendant par là un nombre bien déterminé mais inconnu. Comme je n'ai pas trouvé dans notre langue un mot revêtant une telle signification, et que je n'ai pas voulu alourdir mon exposé en utilisant deux mots pour rendre ce sens, ni forger un mot nouveau dans [notre] langue, je l'ai appelé 'carré' $\left(m e r u b b a^{\prime}\right)$, puisqu'il en est ainsi. Le produit d'un carré par lui-même, ils l'appellent censo di-censo, aussi je l'appellerai 'carré du carré'. Le nombre cubique, ils l'appellent cubo, et le 'cube du cube' cubo di-cubo. Quant aux unités numériques, [ils les appellent] numeri, comme ils le font partout ailleurs.

Après cette introduction, j'étudierai les radicaux; leur connaissance est nécessaire et doit précéder les règles du calcul de l'algèbre (limmudey heshbon ha-alzibra) [...].7

La première partie de l'ouvrage est en effet consacrée aux règles de calcul sur les radicaux, accompagnées de "preuves" géométriques: produit de racines $n$-ièmes $(n=2,3,4)$, de quantités binomiales; somme, quotient, différence. Avant de passer à l'exposé de quinze types d'équations, l'auteur mentionne une prémisse indispensable pour la suite, qu'on peut transcrire ainsi: $x^{4} / x^{3}=x^{3} / x^{2}=x^{2} / x=x / 1$.

Voici la liste des types d'équations traités:

(1) $a x=b$, (2) $a x^{2}=b$, (3) $a x^{2}=b x$, (4) $a x^{2}=b x+c$,

(5) $a x^{2}+b=c x,(6) a x^{2}+b x=c$, (7) $a x^{3}=b$, (8) $a x^{3}=b x$,

(9) $a x^{4}=b$, (10) $a x^{4}=b x$, (11) $a x^{4}=b x^{2}$, (12) $a x^{4}=b x^{3}$, (13) $a x^{3}+b x^{2}=c x$, (14) $a x^{3}+b x=c x^{2},(15) a x^{2}+b x=c x^{3}$.

On relèvera d'emblée l'absence de l'équation $a x^{3}=b x^{2}$, qu'on s'attendrait à trouver entre les équations 8 et 9 , compte tenu de la composition de cette liste. Moṭot a-t-il simplement copié un texte ne faisant pas état de cette équation? l'a-t-il "oubliée"? Voilà qui pose la question de son niveau de compétence mathématique. Nous y revenons.

Les équations 1-5 sont suivies d'un exemple numérique; les équations 4-6 sont accompagnées d'une preuve géométrique. Les équations 7-15 sont réductibles, soit à l'extraction d'une racine, soit à des équations de degré au plus égal à 2 .

L'exposé, dont nous ne commenterons pas le détail, s'achève sur cette péroraison:

7 MS Mantoue, fol. 122b, 1l. 1-18. 
Voilà ce que j’ai cherché et trouvé, par-ci par-là, en matière de calculs du 'livre de l'algèbre', dans les ouvrages des chrétiens. Bien de ces règles, je les ai forgées moi-même. Il faut que tu saches, mon très cher frère, très honorable maître Mordekhay [...], fils de notre honorable maître, Abraham Finzi - que sa mémoire soit en bénédiction dans le monde à venir $-{ }^{8}$ que l'auteur du livre a donné toutes ces règles sans démonstration, dans son ouvrage; et il n'y a pas un sur mille de ceux qui étudient l'ouvrage qui comprenne la démarche suivie par le savant et la manière dont elles [ces règles] ont été établies.

Quant à moi, ton frère, voyant que toi, ainsi que le plus cher de mes compagnons, maître Juda, fils de notre honorable maître Joseph - que Dieu le garde et prolonge sa vie -, fils de notre maître l'honorable Abigdor - que sa mémoire soit en bénédiction dans le monde à venir -, manifestiez un vif désir de connaître l'ouvrage, - or, connaître, au sens où nous l'entendons vraiment, requiert que l'on acquière la connaissance par la voie du syllogisme démonstratif -, moi, pour répondre donc à votre désir, je me suis imposé de réfléchir aux preuves et de les rédiger à votre intention. Toutefois, j'en ai traité brièvement, et cela pour deux raisons: la première, c'est que je fais confiance à votre excellent esprit esprit divin dominant la science; la deuxième concerne les nombreux soucis qui ont assailli mon esprit et mon corps, suite aux mésaventures que j'ai subies, et à toutes les roueries des affaires mondaines [...].

J'intercède auprès de Dieu, qu'Il comble tous tes vœux, que se répandent tes fontaines, fontaines de salut, Amen, selon ta volonté et selon la volonté de ton frère affectionné et dévoué, Simon, fils de notre honorable maître Moïse - que Dieu le garde et prolonge sa vie -, fils de notre maître, l'honorable Simon Moțot - que sa mémoire soit en bénédiction dans le monde à venir. ${ }^{9}$

Quelles indications historiques, utiles pour notre propos, pouvons-nous retirer de ces lignes? Les unes concernent les sources que l'auteur a exploitées pour composer cet opuscule; les autres ont trait à l'intérêt pour l'algèbre qu'aurait manifesté Mordekhay Finzi (à quelle date?) et qui aurait conduit l'auteur à réaliser ce travail (achevé après 1446) à l'intention de Finzi et d'un autre savant.

À l'évidence, les sources de Simon Motot sont italiennes. L'ouvrage qu'il a exploité est un trattato d'abbaco parmi les

\footnotetext{
${ }^{8}$ Cette formule de bénédiction permet d'affirmer que le père de Mordekhay Finzi était décédé au moment où ce texte est écrit. Steinschneider a pu établir qu'Abraham Finzi est effectivement mort en 1446; il relevait qu'une telle date était ainsi la seule indication permettant de fixer un élément biographique de Simon Moțoț, auteur de notre texte: il était actif après 1446; voir M. Steinschneider, Letteratura Italiana dei Giudei (Rome, 1884), p. 52.

${ }^{9}$ MS Mantoue, fol. 132b-133a. Cette conclusion, ainsi que le colophon, ne figurent pas dans le manuscrit d'Amsterdam, lequel comporte plusieurs omissions par rapport aux quatre autres copies.
} 
dizaines de traités de même genre composés entre $\mathrm{XIII}{ }^{\mathrm{e}}$ et $\mathrm{XV}^{\mathrm{e}}$ siècle. ${ }^{10}$

Il est plus difficile de préciser les auteurs ou les livres qu'il a consultés "de ci, de là" pour enrichir son exposé, par exemple ceux auxquels il a emprunté les règles relatives aux radicaux ou les preuves géométriques des équations 4-6. ${ }^{11}$ Soulignons toutefois ceci: les trois preuves géométriques, telles qu'elles sont exposées par Moṭoț, témoignent d'une telle confusion qu'on peut se demander s'il a bien compris les sources qu'il a exploitées. ${ }^{12}$ Si l'on ajoute que Moṭoț, lorsqu'il expose l'équation (5) $a x^{2}+b=c x$, ne mentionne ni le cas de deux solutions possibles, ni celui de l'absence de solution, cas dûment analysés par exemple par Fibonacci, on conclura que Moṭt manifeste un niveau mathématique plutôt faible.

10 En confrontant la liste des équations traitées par Moțot à celle qui figure dans les ouvrages italiens qui nous sont connus, nous pouvons suggérer une source possible: un traité anonyme composé à Lucques (Lucca) en Toscane au XIV $^{\mathrm{e}}$ siècle, le Libro d'abaco. Dans la partie algébrique de l'ouvrage, intitulée Regola della cosa, on trouve à une exception près l'ensemble des quinze équations de Moțoț (le texte italien résout en effet $a x^{3}=b x^{2}$, absente de la liste de Moțoț); les exemples numériques 1 et 5 sont identiques, les exemples 2 et 3 sont semblables; l'ordre est le même pour les équations 1-8, différent pour les équations 9-15. Voir: Scuola Lucchese, Libro d'abaco, Dal Codice 1754 (sec. XIV) della Biblioteca Statale di Lucca; a cura e con introduzione di Gino Arrighi (Lucca, 1973), pp. 108-11. Soulignons-le: nous n'affirmons pas que cet ouvrage est "la" source de Moțoț; d'autres trattati d'abaco existent qui reproduisent, avec de menues différences, des listes d'équations analogues. On relèvera de plus que l'expression "algèbre", quelle qu'en soit l'orthographe, n'apparaît pas dans l'ouvrage anonyme de Lucca.

${ }^{11}$ Ce type de preuves est généralement ignoré de la tradition abaciste, du moins telle que nous la connaissons. La possibilité d'un recours direct à des sources arabes étant exclue, Moțoț aurait pu les trouver dans le Liber Abaci de Fibonacci, comme le suggère Sacerdote; il a pu aussi disposer, en hébreu, de l'ouvrage de géométrie d'Abraham Bar Hiyya, voire de sa version latine le Liber embadorum (1145). (Sur Bar Hiiyya et l'algèbre, on se reportera à: Lévy, “L'algèbre dans les textes hébraïques [I]", pp. 274-6).

${ }^{12}$ Qu'on en juge: dans l'examen de l'équation (4), soit $a x^{2}=b x+c$ (Sacerdote, "Le livre de l'algèbre", vol. 29, pp. 112-13), après avoir indiqué la procédure générale, Moțot propose une preuve géométrique. Comme il ne le précise pas, on devine qu'il s'agit de l'équation $x^{2}=8 x+20$. Il commence par construire un segment $A B$ de longueur 10 ("une ligne de dix mesures [middot]") sur lequel il construit un carré $A B C D$; sur $A B$, il prend le point $Z$ tel que $A Z$ soit de longueur 8; la parallèle $Z H$ à $A C$ et $B D$ complète la figure, de façon à obtenir un carré dont la surface sera égale à celle du rectangle de surface $8 x$ augmenté du rectangle de surface 20, $x$ (la "chose") étant la longueur, recherchée, du côté $A B$ du carré. Mais $A B$, le côté du carré, a déjà été posé comme ayant pour longueur "dix mesures". On ne sera donc pas étonné de trouver à la suite du développement, classique, invoquant la proposition II,6 des Éléments d'Euclide, le 
Enfin, on eût aimé en savoir plus sur le "vif désir" manifesté par Finzi et son collègue (inconnu d'autre part) pour l'ouvrage présenté par Moțoț.13 Leur intérêt et leur curiosité pour l'algèbre étaient-ils déjà éveillés? si oui, par quoi? Du reste, s'agit-il vraiment d'un ouvrage particulier? ou bien "le livre de l'algèbre" désigne-t-il ici de façon générique les règles de base de cette discipline, connue encore seulement par ouï-dire par ses correspondants?

À ces questions, nous n'avons pas de réponse, si ce n'est - très indirectement - les éléments de la biographie de Finzi, en particulier avant 1446 .

\section{MORDEKHAY FINZI, ABŪ KĀMIL ET MAESTRO DARDI DE PISE}

La généalogie des Finzi permet d'attester la présence de cette famille dans le nord de l'Italie dès la deuxième moitié du XIV siècle. Leur activité professionnelle (banquiers, médecins) semble avoir assuré leur prospérité, et sans doute encouragé leur goût des livres: Abraham, le père de Mordekhay, laisse à sa mort (1446) une maison contenant quelque deux cents manuscrits (inventoriés en 1454), ce qui représente une bibliothèque d'une exceptionnelle richesse. Mordekhay est lui-même signalé comme banquier à Mantoue en 1435, activité qu'il semble avoir poursuivie jusqu'à sa mort, entre septembre et décembre $1475 .{ }^{14}$

Dans le domaine scientifique, il a copié, traduit, commenté des textes de divers genres, principalement en astronomie, astrologie, mathématiques; ses écrits originaux, pour être de

bon résultat: $x=10$ ! Le lecteur, même averti, aura eu du mal à évaluer l'intérêt de ladite preuve géométrique. Mais Moṭoṭ lui-même, qu'en a-t-il compris?

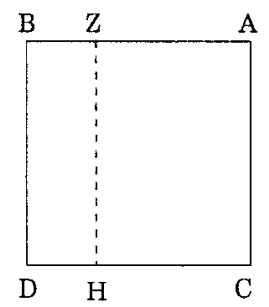

13 “[...] en voyant que [vous] manifestiez le vif désir de 'le' connaître". Mais Moțoț se réfère-t-il vraiment à un ouvrage particulier?

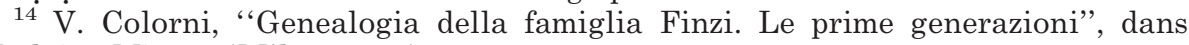
Judaica Minora (Milan, 1983), pp. 329-42. 
moindre portée, ne sont pas négligeables. ${ }^{15}$ Les premières traces de cette activité remontent à 1441: à cette date, il copie entre autres les Éléments d'Euclide, ${ }^{16}$ et traduit du latin, "avec l'aide d'un chrétien", les tables astronomiques de John Batecombe d'Oxford (1348), qu'il adapte aux coordonnées de Mantoue. ${ }^{17}$

Son dernier travail semble être la traduction, à partir de l'italien, de l'imposant traité d'algèbre de Maestro Dardi de Pise (1344) qu'il entreprend en 1473; peut-être la mort a-t-elle interrompu ce travail, car le texte n'en est pas complet. Auparavant, à une date indéterminée, il met au point une version hébraïque de l'Algèbre d'Abū Kāmil, et d'un court texte reprenant la présentation des six équations canoniques figurant dans le même ouvrage. Les textes originaux (respectivement en italien, et en arabe) étant connus, nous nous attacherons surtout à l'analyse de la diffusion des livres et des idées algébriques dont témoignent ces versions hébraïques.

\section{L'Algèbre d'Abū Kāmil: quelles sources Finzi a-t-il exploitées?}

On sait l'importance d'Abū Kāmil (ca. 850-930) dans l'histoire de l'algèbre arabe, une génération après al-Khwārizmī. ${ }^{18}$ Son Algèbre comporte trois parties: (1) un exposé d'al-jabr wa-almuqābala, qui suit l'ouvrage d'al-Khwārizmī tout en le développant par plus d'un trait; (2) une application de l'algèbre à l'étude du pentagone et du décagone réguliers et de quelques problèmes associés; (3) un exposé d'analyse indéterminée.

La tradition manuscrite arabe ne connaît qu'une unique copie du texte complet, datée du XIII ${ }^{\mathrm{e}}$ siècle. ${ }^{19}$ En latin, nous ne

${ }^{15}$ Y. T. Langermann, "The scientific writings of Mordekhai Finzi”, Italia, VII (1988): 7-44.

${ }^{16}$ P. Riccardi, "Sopra un codice ebraico contenente alcuni scritti matematici ed astronomici", Bibliotheca Mathematica, VII (1893): 54-6. Ce codex, longtemps introuvable, vient d'être identifié à Rome; voir G. Lacerenza, "A rediscovered autograph manuscript by Mordekhay Finzi”, Aleph, 3 (2003): 301-25.

${ }_{17}$ Langermann, "The scientific writings of Mordekhai Finzi", pp. 26-7.

${ }^{18}$ R. Rashed, "L'algèbre" et "Analyse combinatoire, analyse numérique, analyse diophantienne et théorie des nombres", dans Rashed (éd.), Histoire des sciences arabes, vol. II, resp. pp. 31-54, 55-91, aux pp. 34-7 et 72-5.

${ }^{19}$ Istanbul, Beyazit Library, MS Kara Mustafa 379; nous en avons utilisé le facsimile: The Book of Algebra by Abu Kämil, Publications of the Institute for the History of Arabic-Islamic Science, Series C, vol. 24 (Francfort-sur-le-Main, 1986). Pour une présentation générale, voir: M. Levey, The Algebra of Abū Kāmil Kitāb al-Jabr wa'l muqūbala in a Commentary by Mordecai Finzi (Madison-Londres, 1966), pp. 7-11; A. Anbouba, "L'algèbre arabe aux IX et X ${ }^{\mathrm{e}}$ 
connaissons qu'une seule copie, incomplète $\left(\mathrm{XIV}^{\mathrm{e}}\right.$ siècle), d'une traduction anonyme: elle comprend les deux premières parties et le tout début de la troisième partie. ${ }^{20}$ On relèvera avec intérêt que le modèle arabe de cette traduction latine comportait plusieurs traces d'intervention en hébreu. ${ }^{21}$

Par contraste, on est surpris par l'abondance relative des témoignages en hébreu. Nous disposons aujourd'hui de deux copies de la version hébraïque associée au nom de Mordekhay Finzi, dont l'une est de la main même de ce dernier, ${ }^{22}$ nous connaissons aussi deux manuscrits transmettant un texte, remanié, de la partie introductive, soit la présentation des six équations canoniques, la section consacrée aux opérations, et l'exposé des "six problèmes" illustrant chaque type d'équations. ${ }^{23}$

siècles. Aperçu général", Journal for the History of Arabic Science, 2 (1978): 66-100, pp. 79-88.

${ }^{20}$ J. Sesiano, "La version latine médiévale de l'Algèbre d'Abū Kāmil”, dans M. Folkerts et J. P. Hogendijk (éds), Vestigia Mathematica. Studies in Honour of H. L. L. Busard (Amsterdam-Atlanta, 1993), pp. 315-452; R. Lorch, "Abū Kāmil on the pentagon and decagon", ibid., pp. 215-52. J. Sesiano a suggéré que l'auteur et copiste de la traduction latine pourrait être Guillaume de Luna, au XIV ${ }^{\mathrm{e}}$ siècle (pp. 315-24); cette suggestion est récusée dans: R. Hissette, "Guillaume de Luna a-t-il traduit Abū Kāmil?"', dans G. Endress et J. A. Aersten (éds), Averroes and the Aristotelian Tradition (Leyde-Boston-Cologne, 1999), pp. 300-15. A. Allard a réuni un ensemble d'indices textuels, minutieusement analysés, qui le conduisent à affirmer qu'une traduction en latin "fut exécutée au plus tard à la fin du XII siècle"; voir Allard, "L'influence des mathématiques arabes dans l'Occident médiéval”, pp. 223-7.

${ }^{21}$ Sesiano, "La version latine médiévale de l'Algèbre d'Abū Kāmil”, p. 322; par exemple: "Scriptum est in Ebraico 'Hic deficit'" (p. 365, 1. 1304).

${ }_{22}$ Mantoue, Biblioteca comunale, MS Ebr. 17, fols. 1a-44b (pour la première partie); 47a-61a (pour la partie sur le pentagone et le décagone); 63b-85b et 112a113b (pour la partie sur l'analyse indéterminée). Tel qu'il nous est parvenu, le texte hébreu s'interrompt avant la fin du texte arabe d'Abū Kāmil, à la page 210 [ = fol. 105b], 1. 13 du fac-similé. L'identification de l'écriture ne fait pas problème; Finzi fut un copiste prolifique et nous disposons aujourd'hui de neuf codex copiés de sa main (Lacerenza, "A rediscovered autograph manuscript by Mordekhay Finzi”, pp. 302-8). Ce manuscrit autographe de Finzi n'était pas connu de M. Levey, dont l'édition repose sur Munich, Bayerische Staatsbibliothek, MS hebr. 225, fols. 95a-191a. M. Levey cite le manuscrit parisien (voir note suivante) en affirmant bien légèrement qu'il transmet le texte du manuscrit de Munich (Levey, The Algebra of Abū Kämil, p. 9): dès les premières lignes, on peut relever les différences, par exemple l'absence d'expressions en espagnol dans le manuscrit parisien.

${ }^{23}$ Paris, BnF, MS hebr. 1029, fols. 296a-309a; Oxford, Bodleian Library, MS Heb. e13 ( = Neubauer 2747), fols. 64a-72b. Rectifions à ce propos une erreur relative au manuscrit parisien, due à l'auteur du catalogue (H. Zotenberg, Catalogue des manuscrits hébreux et samaritains de la Bibliothèque impériale [Paris, 1866], p. 187) et reprise ensuite par les divers bibliographes: à cause du 
La première partie de la version hébraïque de l'ouvrage d'Abū Kāmil, soit l'exposé algébrique proprement dit et les problèmes qui l'accompagnent, a été traduite en allemand par J. Weinberg (1935), ${ }^{24}$ en anglais par M. Levey (1966). ${ }^{25}$ La deuxième partie du texte hébraïque, soit les vingt problèmes de géométrie traités par la voie algébrique, a été traduite en italien par Sacerdote (1896). ${ }^{26}$ Quant à la troisième partie de cette version hébraïque, consacrée pour l'essentiel à l'analyse indéterminée, elle a été "comparée" par M. Levey et P. Schub au texte arabe dont ils ont offert une traduction en anglais. ${ }^{27}$

En l'absence d'une édition scientifique du texte arabe, il est vain de tenter d'évaluer les qualités et les défauts de la traduction. Mordekhay Finzi n'est pas un mathématicien professionnel: certaines de ses gloses portant sur le contenu mathématique en témoignent; en revanche les gloses d'ordre textuel retiendront l'attention de l'historien. Nous avons analysé les deux familles de textes hébraïques en tentant de répondre aux questions suivantes:

titre donné par le copiste (Le livre d'Abū Kāmil sur les procédés ingénieux relatifs au nombre), Zotenberg a suggéré que l'original arabe en était le Kitāb al-Ṭarā'if fī al-hisāb (Le livre des choses rares dans le calcul), que nous connaissons d'autre part; il n'en est rien.

${ }_{24}^{2}$ J. Weinberg, Die Algebra des Abū Kāmil Shogā‘ ben Aslam (Munich, 1935). À l'époque de son travail, Weinberg ignorait l'existence d'une copie du texte arabe.

${ }_{25}$ Levey, The Algebra of Abū Kămil. Le texte hébreu édité est, on l'a dit, celui du manuscrit de Munich; on doit désormais le compléter, parfois le corriger, grâce au manuscrit autographe de Finzi; la traduction anglaise n'est pas sans défauts: M. Levey s'éloigne parfois du texte hébreu, sans le signaler; enfin, disons-le sans détour, les remarques introductives consacrées aux sources exploitées par Finzi n'ont aucune valeur, ni historique, ni méthodologique (pp. 12-13). Les interrogations que Steinschneider avait formulées en son temps restent toujours pertinentes; tout en les signalant, M. Levey n'en a pas tenu compte. Voir M. Steinschneider, Die Hebräischen Übersetzungen und die Juden als Dolmetscher (Berlin, 1893; réimpr. Graz, 1956), pp. 584-7.

${ }^{26}$ G. Sacerdote, "Il trattato del pentagono e del decagono di Abu Kamil Shogia“ ben Aslam ben Muhammed", dans Festschrift zum Achtzigsten Geburtstage M. Steinschneider's (Leipzig, 1896; réimpr. Hildesheim / New York, 1975), pp. 169-94. En l'absence d'un texte arabe, Suter a proposé "une version" allemande de cette partie, à partir de la traduction italienne du texte hébreu: $\mathrm{H}$. Suter, "Die Abhandlung des Abū Kāmil Shogā' b. Aslam 'über das Fünfeck und Zehneck'”, Bibliotheca Mathematica, 10 (1909/10): 15-42. Une version anglaise du texte arabe de cette partie est proposée dans: M. Yadegari et M. Levey, "Abū Kāmil's 'On the pentagon and decagon'", Japanese Studies in the History of Science, $\mathrm{n}^{\circ}$ Suppl. 2 (1971): 1-54; nous n'avons pas eu accès à cette publication.

${ }_{27}$ P. Schub et Martin Levey, "Indeterminate problems of Abū Kāmil (850-930)", dans Atti Della Accademia Nazionale Dei Lincei, Memorie, Classe di Scienze fisiche, matematiche e naturali, Serie VIII, Volume X, Fascicolo 2 (Rome, 1970), pp. 23-96. Reconnaissons-le: cette traduction n'est pas toujours satisfaisante. 
1. quelle est la source (ou les sources) dont dérive le texte hébreu principal? comment rendre compte des nombreuses expressions en castillan qu'il contient? quel a été le rôle exact de Mordekhay Finzi, généralement présenté comme le traducteur?

2. quel est le statut du "petit" texte, que transmettent les manuscrits de Paris et d'Oxford, et qui est aussi associé au nom de Finzi?

Nous suggérons les conclusions suivantes:

1. Finzi n'a pas traduit de l'arabe en hébreu le texte d'Abū Kāmil: il en a révisé et glosé une version hébraïque; cette dernière a été réalisée à partir de deux sources: une source arabe et une source espagnole. Nous ne savons rien du traducteur de la source arabe, si ce n'est qu'il s'agit sans doute d'un juif hispanophone. Quant à la possibilité d'une version espagnole (suggérée avec prudence par Steinschneider, puis par Sacerdote), elle nous paraît se dégager de certains traits du texte hébreu copié par Finzi. S'interroger sur les possibles sources de cette probable version espagnole serait hors de propos.

2. Le "petit" texte, on l'a dit, est une rédactionremaniement de la partie introductive du texte d'Abū Kāmil. La langue et la terminologie sont plus unifiées que dans le texte hébreu principal. Finzi intervient, parfois longuement, comme glossateur; il nous paraît être l'auteur de ce remaniement.

Détaillons maintenant les indices textuels qui nous inclinent, dans l'état actuel de la documentation, à proposer ces conclusions.

\section{Termes, locutions, phrases et gloses en castillan}

Sans entrer dans le détail, nous pouvons affirmer que le manuscrit de Munich (désigné sous le sigle MN) a été copié sur celui de Mantoue (désigné sous le sigle MT), lequel est de la main de Finzi. Certaines des variantes qui les distinguent semblent indiquer que le copiste de MN ne comprenait plus guère le sens des mots espagnols.

MT abonde en expressions espagnoles. En voici quelques exemples significatifs: 
- $\operatorname{algo}(s)$ pour désigner le bien / le carré, soit $m \bar{a} l$ en arabe, census en latin;28

- titulo $(s)$ pour désigner une des "deux voies" de résolution, celle qui conduit à la racine, et celle qui conduit au carré; en arabe $b \bar{a} b \bar{a} n$, en latin due vie $;^{29}$

- l'expression al-jabr wa-al-muqābala est translittérée en caractères hébraïques, et elle est suivie par une traduction en castillan cobramiento e confrontamiento, ${ }^{30}$ qui rend bien le sens du doublet arabe et qui revient fréquemment dans le texte. Les verbes dont dérivent ces substantifs, cobrar et confrontar, apparaissent souvent dans les gloses où ils sont parfois accompagnés par d'autres termes hébreux que ceux qui figurent dans le texte: on a le sentiment que le glossateur (Finzi, à notre avis) tente ainsi d'unifier la terminologie face aux hésitations du traducteur. ${ }^{31}$ Nous en avons d'autres indices. $^{32}$

${ }^{28}$ En marge interlinéaire (MT, fol. 1a, 1. 2), le mot 'shorashim/racines' est désigné comme radis, et le mot 'misparim / nombres' par cuentas. Toutefois, seul le terme $\operatorname{algo}(s)$ sera ensuite utilisé systématiquement.

${ }^{29}$ MT, fol. 1 b, 1l. 15, 18, 22. Ce terme de castillan médiéval peut signifier 'tête de chapitre'; le choix du traducteur aurait-il été inspiré par l'un des sens du mot arabe ' $b \bar{a} b$ '? Quoi qu'il en soit, le terme castillan est remplacé dans la suite du texte par le terme hébreu ofen (aspect, voie, méthode), plus familier à un lecteur non hispanophone. MN ne reproduit pas le terme castillan.

${ }^{30}$ MT, fol. 6b; MN, fol. 102b (Levey, p. 53).

31 Dans les trois exemples suivants, le verbe arabe issu de la racine j.b.r. est rendu par trois verbes hébreux différents, pourtant glosés par le même verbe castillan cobrar.

Dans le problème $\mathrm{n}^{\mathrm{o}} 1$, on considère l'égalité $x^{2}=15 x-1 \frac{1 / 2}{2}$; on lit alors: "isole (tanniah) les quinze [racines] par rapport [au terme] un bien et demi, de façon qu'ils deviennent 15 racines [seules], et ajoute le bien et demi au bien; il vient alors deux biens et demi égalent 15 racines [...]"; une glose marginale, mêlant l'espagnol et l'hébreu, indique “e-cobrias, ke-lomar te'esof (héb.: c'est-à-dire rassemble)" (MT, fol. 13b).

Dans le problème $\mathrm{n}^{\circ} 3$, le même verbe arabe est rendu par 'fais un tout (tikhlol)', qui est aussi glosé par 'e-cobrias' (MT, fol. 14b).

Dans le problème $\mathrm{n}^{\circ} 4$, on relève encore un autre verbe hébreu 'recouvre-les (tașși 'am )', qui est pareillement glosé 'e-cobralos', suivi à nouveau d'une variante en hébreu 'we-te'espam (et rassemble-les)', utilisant le même verbe que celui qui accompagne la première glose (MT, fol. 14b).

On relèvera avec intérêt que dans le "petit" texte, dans les passages correspondants (problèmes 1, 3 et 4, voir Paris, MS hebr. 1029, fol. 307a et 307b), la terminologie est unifiée: un seul et même verbe hébreu est utilisé, celui qui figure dans les gloses citées ci-dessus, asaf. Nous avons relevé dans d'autres gloses du texte principal la présence de ce terme hébreu (asaf), accompagnant la mention du terme espagnol cobrar (MT, fol. 16a, 51a).

${ }^{32}$ Dans une glose, clairement précédée de ses initiales (a.m.f., soit amar Mordekhay Finzi, Mordekhay Finzi a dit), Finzi commente, de manière assez 
Les expressions en espagnol ne concernent pas seulement la terminologie technique, mais aussi des mots courants et quelquefois, ce qui est plus étrange, des tronçons de phrases. Dans ce cas, il semble bien que le rédacteur du texte hébreu, perplexe face à la phrase arabe, se soit contenté d'un prudent mot à mot espagnol, ou alors, ait emprunté le passage à une source espagnole. ${ }^{33}$

\section{Une source espagnole}

Comment interpréter la présence de ces nombreuses expressions espagnoles, qui sont encore fréquentes dans les deuxième et troisième parties de l'ouvrage ${ }^{34}$ Leur nombre et leur diversité suffiraient à conjecturer l'existence d'une source

vague, le terme confrontamiento: “Rappelle-toi que ceci, c'est 'l'appariement (l'ajustement)' (ha-kiwwun), qui est toujours mentionné en langue étrangère (be-la'az) comme confrontamiento, car, par là [par cette opération], on vise à obtenir (yakhwin) ce que vaut la chose [...]" (MT, fol. 16b). De fait, dans le "petit" texte (celui que transmettent les manuscrits de Paris et d'Oxford), Finzi affirme avoir forgé lui-même le terme kiwwun, pour traduire le mot, désigné "en langue étrangère", (dans ce cas, en castillan) par confrontamiento. Voir, plus loin, n. 46 .

${ }^{33}$ Un exemple significatif. On répartit 10 en deux parties $(x$ et $10-x)$ et on divise 10 par chacune d'elles; la somme de ces deux quotients est $61 / 4$. Il s'agit de montrer que la somme des quotients de chacune des deux parties par l'autre est égale à la somme précédente diminuée de 2 , soit $41 / 4$. Avec notre écriture moderne des fractions, il est aisé de vérifier que $10 / x$ peut s'écrire $[(10-x) /$ $x]+1$, et que $10 /(10-x)$ peut s'écrire $[x /(10-x)]+1$; il en résulte la propriété énoncée. À l'époque d'Abū Kāmil, la manipulation de ces fractions est moins simple et ne s'exprime que rhétoriquement. Avant de donner une preuve par la "méthode géométrique (tarīq al-handasa)", c'est-à-dire utilisant des segments, le savant arabe expose la "méthode du signe (tarīq al-ishāra)", celle qui exploite le fait que la somme du dividende (numérateur) et du diviseur (dénominateur) de chacune des deux fractions $(10-x) / x$ et $x /(10-x)$ est toujours égale à 10 . Il écrit: "La cause de ce qui est dit à propos de 2 est avérée par la méthode du signe [...]" (Abū Kāmil, Kitāb al-Jabr, fac-similé, p. 125, 1. 9). Le traducteur latin rendra cette phrase littéralement: "Et causa in 2 vera ex via signi est [...]" (Sesiano, “Algèbre d'Abū Kāmil”, p. 413, 1. 3294). Le rédacteur du texte hébreu a manifestement du mal à identifier ce dont il s'agit précisément, ou alors se sent incapable de trouver une formulation en hébreu; aussi il commence sa phrase en hébreu, pour la finir par un mot à mot espagnol: "La raison de cette différence de 2 (sibat zeh ha-gera'on ha-shenayim) sana $i$ son levantara di parti di las sinias [...]" (MT, fol. 44b, MN, fol. 150a, Levey, p. 203) où l'on peut reconnaître, non sans quelque effort, le mot à mot de la fin de la phrase arabe. Pour autant, le lecteur hébréophone aura-t-il compris, s'il n'est pas familier du parler roman-castillan?

${ }^{34}$ Sacerdote, qui a traduit en italien la deuxième partie, était ainsi conduit à suggérer, prudemment il est vrai, une source espagnole à la traduction hébraïque (Sacerdote, "Il trattato del pentagono", p. 176). Du reste, plusieurs expressions suscitaient sa perplexité; ainsi Sacerdote n'a pas compris l'expression, 
espagnole. Mais il y a plus: Finzi lui-même fait état, au moins une fois, d'un tel ouvrage lorsqu'il mentionne "le livre en langue étrangère". Cette glose de Finzi apparaît à la fin d'un problème de géométrie, face auquel il avoue sa perplexité: déterminer la longueur du pentagone équilatère inscriptible dans un carré de côté 10.

Arrêtons-nous sur l'exemple; 35 l'embarras qu'il suscite chez Finzi nous vaut une longue remarque de ce dernier, laquelle a

translittérée en caractères hébraïques "por lo mas circano", qui traduit, tout simplement, l'arabe "bi-al-taqrīb", soit "de manière approchée" (ibid. p. 186, n. 1; pour l'arabe: Abū Kāmil, Kitāb al-Jabr, fac-similé, p. 146, 1. 11). Grâce au texte arabe et à l'autographe de Finzi, nous pouvons résoudre ce type de difficultés.

${ }_{35}$ Abū Kāmil, Kitāb al-Jabr, fac-similé, p. 150, 1. 2-12; Héb., MT, fol. 58a-b, $\mathrm{MN}$, fol. 162a-b (la traduction italienne comporte quelques erreurs de lecture: Sacerdote, "Il trattato del pentagono e del decagono", pp. 188-9); latin, Lorch, "Abū Kāmil on the pentagon and decagon", pp. 245-6. Il n'est pas sans intérêt d'examiner la transmission des figures géométriques:

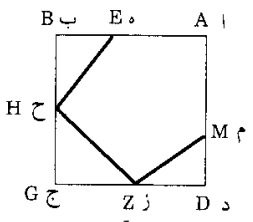

arabe

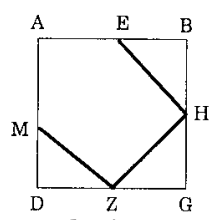

latin

Dans ce problème (le quinzième), l'auteur se donne un carré de côté $10, A B G D$; il y inscrit "un pentagone" (sans autre précision), selon une figure accompagnant le texte, $A E H Z M$, et se propose de déterminer "chacun de ses côtés". À cette fin, il pose l'un des côtés, soit le côté $A E$, égal "à une chose" $(x)$; la mise en équation traduit bien qu'il ne peut s'agir que d'un pentagone équilatère, non équiangle (sur la figure du texte arabe ainsi que du texte latin, l'angle $A$ du pentagone n'est autre que l'angle $A$ du carré, soit un angle droit! La figure du texte hébreu est, en revanche, passablement confuse); elle conduit à l'égalité: $200+1 \frac{1}{2} x^{2}-20 x-$ $\sqrt{ }\left[200 x^{2}\right]=x^{2}$. L'auteur appelle le lecteur à procéder par muqābala, comme je te l'ai indiqué [...]", et donne, sans détailler, le résultat recherché, "le côté $A E$, soit l'un des côtés du pentagone": $x=20+\sqrt{ } 200-\sqrt{ }[200+\sqrt{320000]}$.

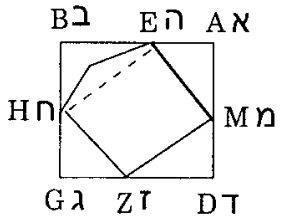

Fig. 1

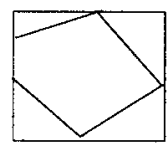

Fig. 2

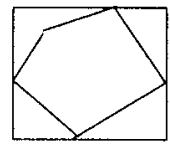

Fig. 3

Le texte hébreu, aussi bref que le texte arabe, comporte de surcroît une lacune de quelques mots, due sans doute à un saut du même au même. Surtout, la figure dont dispose Finzi ne semble guère l'avoir aidé à comprendre le problème: il pense qu'il s'agit d'inscrire dans un carré un pentagone équilatère et équiangle, problème qu'il finit, à juste titre, par reconnaître comme impossible; d'où son embarras. Mais a-t-il bien compris le texte, dont la traduction en hébreu est pourtant assez fidèle, malgré la lacune signalée? 
retenu notre attention. Voici la glose, insérée dans le texte hébreu, à la suite du problème:

Moi, Mordekhay le rédacteur (ha-kotev), j'ai copié (he'taqti, que nous rendons par "copié" plutôt que par "traduit", qui est aussi lexicalement possible) mot à mot ce que j'ai trouvé, mais je n'ai rien compris à cette figure [la première des trois figures accompagnant le texte hébreu]. Il me semble que la proposition à établir concerne aussi [comme dans quelques-uns des problèmes précédents] un pentagone équilatère et équiangle, circonscrit par le carré, comme dans cette deuxième figure. Après que j'ai essayé de dessiner cette deuxième figure, j'ai compris que le quadrilatère circonscrivant le pentagone [équilatère et équiangle] est un rectangle et non un carré: il m'apparaît, en effet, impossible de circonscrire un véritable carré autour du pentagone équilatère et équiangle, si ce n'est comme dans cette troisième figure, dont les contours sont disposés comme les contours de la première figure, celle qui a été copiée du livre en langue étrangère (asher ne'teqa min ha-sefer ha-la'az).

J'ai bien cru, au début, que la figure, dans le livre, était erronée, et je me disposais à la corriger, comme je l'ai fait pour de nombreuses autres figures.

Dieu soit loué, qui m'a ouvert les yeux, de même qu'Il m'a fait voir les merveilles de Sa Tora. Amen, qu'il en soit ainsi.

Que tirons-nous de cette remarque?

D'abord que Finzi n'a pas lu attentivement le texte même qu'il transcrit, lequel indique bien qu'on part d'un carré (de côté 10) avant d'y inscrire un pentagone: la mise en équation ne laisse aucun doute à cet égard; la seule caractéristique exploitée est celle de l'égalité des cinq côtés dudit pentagone, et en aucune manière l'égalité des angles. Il est vrai que la figure que reproduit Finzi est pour le moins confuse [Fig. 1]. L'embarras que manifeste Finzi nous apprend - et cela est précieux - que la première figure a été reproduite "à partir du livre en langue étrangère (la' $a z)$ ". ${ }^{36}$ Dans notre texte, l'expression la' $a z$ accompagne fréquemment les gloses ou les termes formulés en castillan, à commencer par le doublet cobramiento e confrontamiento. Ajoutons que l'expression $l a$ ' $a z$ ne désigne jamais la langue arabe dans les textes médiévaux européens: il s'agit, en général, d'une langue vernaculaire (identifiable selon le contexte), plus rarement le latin; par exemple le français-champenois, au $\mathrm{XI}^{\mathrm{e}}$ siècle, sous la plume du célèbre commentateur biblique et talmudique de Troyes Rabbi Salomon ben Ishạaq (Rashi).

\footnotetext{
${ }^{36} l a ' a z$, acronyme de "leshon 'am zar", qu'une étymologie populaire fait
} remonter à l'expression biblique "un peuple étranger, 'am lo'ez" (Psaumes, 114,1). 
Nous pouvons donc affirmer que Finzi fait état, ici, d'un livre rédigé en castillan. De surcroît, contrairement à l'usage qui est le sien (avéré plusieurs fois dans le texte), Finzi ne dit pas "[dans] le livre à partir duquel j'ai copié", mais "[la figure] qui a été copiée du livre en langue étrangère". La confusion même de la figure reproduite par Finzi (sans doute fidèlement) nous paraît résulter d'un mélange de deux figures, l'une orientée de droite à gauche (comme en arabe) et l'autre de gauche à droite (indice d'une copie en espagnol?).

Il semble avéré que Finzi connaît l'existence d'un texte espagnol (nous ne savons pas s'il en a disposé) et que celui-ci a été exploité dans l'élaboration du texte hébreu qu'il nous offre.

Doit-on conclure que le texte hébreu dont nous disposons est une traduction faite à partir de ce texte espagnol? Même si on ne peut exclure cette hypothèse, elle ne rend pas compte de toutes les caractéristiques du document que nous a transmis Finzi.

Plusieurs indices nous paraissent attester aussi un recours direct à un texte arabe:

- phrases hébraïques dont la syntaxe épouse la syntaxe des phrases arabes;

- lettrage des figures géométriques suivant fidèlement celui des figures du texte arabe (en particulier, dans l'usage de la consonne gutturale 'ayin, qui se retrouve dans le texte hébreu à la place où elle figure dans le texte arabe, ce qui serait invraisemblable si l'hébreu était issu de l'espagnol seulement);

- contre-sens issus d'une lecture erronée de mots arabes (induite sans doute par l'absence de points diacritiques dans le modèle arabe);

- translittération de certains mots arabes. ${ }^{37}$

Concluons: selon toute vraisemblance, une source arabe et une source castillane ont été exploitées dans l'élaboration du texte hébreu.

${ }^{37}$ Dans ces deux derniers cas, on peut toujours nous objecter que le phénomène a pu intervenir dans le passage de l'arabe au castillan, et n'implique pas nécessairement un passage direct de l'arabe à l'hébreu. Il appartiendrait à des philologues plus compétents d'interpréter, par exemple, la translittération du mot arabe juljulān (sésame) en caractères hébraïques (MT, fol. 113a) j.w.l.j.w.l.'.n/ et de se demander si un intermédiaire roman-castillan est possible. 


\section{Finzi n'a pas traduit}

Nous affirmons que Finzi n'est pas l'auteur de la traduction hébraïque que nous lisons.

Nous pouvons écarter rapidement l'hypothèse (retenue par Levey) d'une traduction directe en hébreu, par Finzi, d'un texte arabe. Dans tout ce que nous savons des écrits de Finzi, il n'existe pas le moindre indice d'une quelconque connaissance de l'arabe. De surcroît, le recours avéré à une source espagnole réduit d'autant la validité d'une telle hypothèse.

Finzi aurait-il traduit un texte espagnol? Dans ce cas, pourquoi aurait-il gardé tant d'expressions, de tournures, de termes espagnols, alors même qu'il lui arrive, dans les gloses qu'il signe, de proposer des termes hébreux différents de ceux qui apparaissent dans le corps du texte? Un autre détail, significatif, nous invite à écarter une telle hypothèse: le texte autographe de Finzi, dans la deuxième partie de l'ouvrage, use d'une tournure bien insolite pour désigner la "corde" d'un cercle, soit "la droite sécante (qaw hotekh)" (dans le texte arabe, watar; dans le texte latin, corda). Finzi qui a traduit et / ou copié des textes astronomiques et mathématiques (sa copie des Éléments d'Euclide date de 1441), connaît parfaitement l'existence du terme hébreu "meytar", qu'il utilise du reste dans ce même codex, dans un ouvrage de stéréométrie, signé de son nom et copié de sa main. ${ }^{38}$

Concluons sur ce point: Finzi est le copiste-réviseurglossateur d'un texte hébreu qui lui est parvenu. L'auteur de ce dernier texte, ainsi que les conditions de la transmission, nous sont inconnus.

\section{Le "petit" texte: une rédaction, due à Finzi?}

Examinons le contenu du "petit" texte. On l'a dit: on y retrouve (dans l'ensemble, mais non dans le détail) la présentation des six équations canoniques, la section consacrée aux opérations, et enfin l'exposé des "six problèmes" illustrant chacun des six types d'équations canoniques. ${ }^{39}$ Le texte est divisé en sections (peraqim), et scandé par les mots "Il a dit".

${ }^{38}$ MT, fol. 118a, 1. 4: “[...] de là, tu connaîtras la corde (meytar) de cet arc $[\ldots] "$. .

${ }^{39}$ Le manuscrit d'Oxford s'interrompt au milieu de l'exposé sur la multiplication (correspondant au manuscrit de Paris, fol. 302a, 1. 15), peut-être à la fin d'un cahier. 
Des gloses, introduites par la mention "Mordekhay Finzi a dit", sont insérées dans le corps du texte.

Le "petit" texte est bien issu d'un remaniement du texte principal, cela ne fait aucun doute. Ayant comparé ligne à ligne ces deux recensions ( $\mathrm{du}$ moins leur partie commune), nous pouvons l'affirmer: le "petit" texte rédige de manière plus unifiée (du point de vue de la langue) le texte principal; l'argumentation et les preuves géométriques sont celles du texte d'Abū Kāmil, mais la formulation, le lettrage des figures et l'ordre des conclusions sont parfois modifiés; de surcroît, on ne relève plus aucune trace d'espagnol, sauf une brève mention du doublet cobramiento $i$ confrontamiento. ${ }^{40}$

Plusieurs exemples attestent de la continuité des deux recensions et nous permettent d'affirmer que le "petit" texte est bien issu du texte principal et qu'il a sans doute pour auteur Finzi lui-même.

Le premier exemple est celui d'un contre-sens, qui a sa source dans une lecture erronée du texte arabe. Le passage concerné intervient après qu'a été exposée et justifiée la double résolution de la sixième équation canonique, celle qui permet d'obtenir la racine, et celle qui aboutit au carré de la racine. Voici le passage tel qu'il figure dans le "petit" texte:

Il a dit: cette partie est achevée, et avec elle s'achève l'explication des six cas, dont nous entendions expliquer les modes d'usage et les preuves afférentes. Nombre d'auteurs (harbeh sofrey) de al-jabr et al-muqābala ne peuvent pas ne pas t'en indiquer (lo yitakhen she-lo yar'ukha) certains d'entre eux [les six cas]. À chacun de ces six cas [correspondent] des problèmes [résolubles] par la mise en dépôt (ha-hafqada) et par l'ajustement ( ha-kiwwun) - en langue étranger̀e (be-la'az) cobramiento $i$ confrontamiento - auxquels te conduisent les arithméticiens (ba'aley ha-mispar).

Mordekhay Finzi a dit: j'ai forgé [le terme] hafqada à partir du mot piqqadon (dépôt, gage), et kiwwun à partir de l'expression kiwwen heshbon (apparier un nombre, l'associer), lesquels figurent dans les propos de nos maîtres, de mémoire bénie. Tu en comprendras le sens dans le cadre des problèmes qu'il présentera, après les propriétés qu'il va exposer maintenant. ${ }^{41}$

Avant de commenter, donnons le passage correspondant dans le texte principal; l'hébreu y est plus fidèle à l'arabe (au même contre-sens près), que nous présentons en vis-à-vis:

\footnotetext{
${ }^{40}$ Le lecteur hébraïsant peut s'en assurer en plaçant en vis-à-vis deux passages, n'importe lesquels, qui se correspondent dans chacun de deux textes.

${ }^{41}$ MSS Paris, fol. 301b; Oxford, fol. 72a.
} 
Voici les six cas (halaqim) que nous avons énoncés dans ce livre et dont nous avons expliqué la résolution (mal'akhatam) et les causes ('illoteyhem). Trois d'entre eux sont simples (nifradim), ce sont [...], et trois en sont composés, ce sont [...]. Nombre d'auteurs (harbeh sofrey) de al-jabr et al-muqābala ne peuvent pas ne pas t'en indiquer (lo yukhal lihyot she-lo yar'ukha) certains d'entre eux [les six cas]. À chacun de ces six cas [correspondent] des problèmes (she'elot) [à la résolution desquels] te conduisent les arithméticiens (ba'aley ha-mispar) par le cobramiento et le confrontamiento [.. .]. ${ }^{42}$
Voici les six cas (al-durāb) que nous avons énoncés dans ce livre qui est nôtre et dont nous avons expliqué la résolution ( $q i y \bar{a} \operatorname{sih} \bar{a})$ et la cause ( $a l$-'illa fīh $\bar{a})$. Trois d'entre eux sont simples, ce sont [...], et trois d'entre eux sont composés, ce sont [...]. Pour l'essentiel, le calcul (kathīran min hisāb) d'al jabr et d'al-muqābala ne peut que (lā budda an) te conduire à l'un d'entre eux [les six cas]. J'ai posé pour chacun de ces six cas un problème [à la résolution duquel] te conduit le calcul d'al jabr et d'al-muqābala $[\ldots] .{ }^{43}$

Un contre-sens manifeste a fait lire au traducteur "les auteurs" ou "les arithméticiens" là où le texte arabe indique sans confusion possible "le calcul". ${ }^{44}$ Le contre-sens, on l'a vu, figure aussi dans le "petit" texte. On relève une autre occurrence de ce contre-sens plus loin, dans chacun des deux textes hébraïques. ${ }^{45}$

Dans le "petit" texte, l'unique mention de termes espagnols figure dans le passage que nous venons de citer; elle est suivie d'une glose de Finzi lui-même qui témoigne de son effort pour unifier la terminologie; Finzi nous indique qu'il a forgé des

${ }^{42}$ MT, fol. 6b; MN, fol. 102b (Levey, p. 53).

43 Abū Kāmil, Kitāb al-Jabr, fac-similé, p. 23.

${ }^{44}$ Le terme arabe hisāb (calcul) peut être lu hussāb (calculateurs). Sur ce point, le traducteur latin, malgré une lourdeur évidente due à son souci de littéralité, ne se trompe pas lorsqu'il écrit: "Et plurimum computationis el geber et el mukabale (glosé: id est restaurationis et oppositionis) [...]" (Sesiano, "Algèbre d'Abū Kāmil'", p. 340, 1l. 526-28).

${ }^{45}$ Là où l'arabe (ainsi que le latin) indique clairement "le calcul de l'algèbre ( his āb al-jabr / computatio restaurationis)" (Abū Kāmil, Kitāb al-Jabr, fac-similé, p. 42, l. 17; Sesiano, "Algèbre d'Abū Kāmil", p. 358, 1. 1060), le texte hébreu principal ainsi que le "petit" texte indiquent "les savants [dans le domaine] de l'algèbre (hakhmey ha-aljabr, le traducteur ayant lu hussāb al-jabr)" (MT, fol. 13b; MN, fol. 111a [Levey, p. 82]; MS Paris, fol. 307a [le manuscrit d'Oxford s'interrompt avant ce passage]). 
termes hébraïques, dérivés de la terminologie rabbinique. ${ }^{46}$ Nous avions précédemment relevé cet effort dans les gloses du texte principal.

Un autre exemple retient notre attention: il concerne une lacune dans le texte principal, que relève, à juste titre, Finzi. ${ }^{47}$ Constatant l'absence d'un argument dans "le livre à partir duquel j'ai copié (ha-sefer asher he'taqti mimmenu)", Finzi ajoute: "j'en donnerai l'explication en hébreu, toujours à

${ }^{46}$ Arrêtons-nous un moment sur l'effort terminologique de Finzi pour "adapter" en hébreu l'expression arabo-castillane cobramiento e confrontamiento; il revêt une portée qui déborde le seul commentaire philologique. Le verbe cobrar, dérivé de recobrar (latin recuperare) est attesté dès le XIII ${ }^{\mathrm{e}}$ siècle au sens de 'recouvrer ce qui est perdu', 'récupérer ce qui a été mis en gage': c'est ainsi qu'on relève, dans le recueil des édits royaux d'Alphonse X le Sage (1255) "cobren lo que dieron en guarda"; voir Fuero Real del Rey Don Alonso el Sabio (Madrid, 1836), p. 101. Dans le premier dictionnaire castillan-arabe, El vocabulario de Pedro Alcala (1501), le terme 'cobrar lo perdido' est référé aux racines arabes h.l.f et g.b.r, et l'on peut lire dans l'entrée ğ.b.r: cobrar [lo perdido], ğabra, "algebra arte"; voir Elena Pezzi, El vocabulario de Pedro de Alcala (Almeria, 1989), s.v. cobrar et $\check{g} . b . r$.

Pour rendre le terme cobramiento, Finzi nous dit avoir forgé le substantif hafqada, à partir du terme piqqadon, 'objet mis en dépôt' (ainsi, dans Lév. 5,23 "[...] le dépôt qui lui a été confié, ha-piqqadon asher hafqad itto"): il nous apparaît que Finzi a été ici plus sensible à un aspect de l'opération al-jabr, à savoir l'objet sur lequel elle porte, qu'à l'opération elle-même, le recouvrement, la restitution (restauratio en latin). Soulignons que ce substantif et le verbe qui pourrait lui être associé ne sont pas utilisés dans le corps du "petit" texte: on s'y sert généralement du verbe asaf pour rendre l'opération al-jabr.

Pour rendre confrontamiento, Finzi nous dit s'être appuyé sur l'expression kiwwen heshbon, 'apparier un nombre' ou 'associer un nombre [à un autre]' (qu'on ne trouve dans aucun dictionnaire, ancien ou moderne) et forgé le substantif kiwwun. Pour comprendre quel sens Finzi confère à cette expression, nous avons essayé d'en localiser des occurrences dans la vaste littérature rabbinique, d'où Finzi nous dit l'avoir tirée. L'expression est fort rare; elle n'est même pas mentionnée dans le classique Thesaurus de E. Ben Yehuda, dans l'entrée 'kiwwen'. Nous l'avons repérée dans le commentaire du livre de Daniel par Rashi ( $\mathrm{XI}^{\mathrm{e}}$ siècle); à propos des calculs relatifs à la fin des temps, le commentaire "associe" les "2300 jours et nuits" (Dan. 8,14) et les "1335 jours" (Dan. 12,11) en écrivant: "ce nombre-ci [1335] est associé au nombre des jours et nuits (heshbon zeh mekhuwwan 'im heshbon 'erev u-voqer), 2300 [...]". Finzi avait déjà glosé, de manière un peu vague, un passage du texte principal en proposant de rendre le terme castillan par l'hébreu ha-kiwwun (voir supra n. 32). Ici, Finzi semble plus proche du sens technique de l'opération muqābala (association des termes semblables).

Par ces remarques, nous voulons souligner ceci: si Finzi avait été en mesure de maîtriser un texte arabe, il aurait sans aucun doute mieux adapté la terminologie hébraïque proposée.

${ }^{47}$ MT, fols. 4b-5a, MN, fols. 100a-100b (Levey, pp. 47-9). Le passage manquant en hébreu figure dans le texte arabe: Abū Kāmil, Kitāb al-Jabr, fac-similé, p. 17, 1. 15-19, 1. 15. Il s'agit, dans le cours de la résolution de l'équation $x^{2}+25=10 x$, de la méthode permettant d'obtenir directement le carré $\left(x^{2}\right)$. 
propos de cet ouvrage, avec l'aide de Dieu (eva'erehu be-'ivri 'od 'al zeh ha-sefer ....)". C'est, en effet, ce qu'il fait dans le "petit" texte. Intervenant dans le même passage, et constatant la même lacune, Finzi écrit "[. . .] dans le livre à partir duquel j'ai copié, la preuve manque relativement à la méthode qui nous donne le carré. C'est pourquoi, j'ai jugé bon de l'expliquer. À cette fin, je tracerai trois figures [. . .]". ${ }^{48}$

Concluons: le "petit" texte est adapté du texte principal; nous inclinons à penser que Finzi en est lui-même l'auteur.

\section{Finzi, traducteur (1473) du traité Algeble amugabala de Maestro Dardi de Pise (1344)}

Présenté à juste titre comme une œuvre importante de l'algèbre italienne au Moyen Âge, le traité de Dardi propose une liste de 198 équations de degré 1 à 4, commentées et résolues. Mieux, il comprend 4 équations cubiques et quartiques irréductibles: ces dernières ne sont pas résolues de façon générale, mais dans des cas numériques particuliers qui permettent de les ramener à des équations cubiques ou quartiques pures. ${ }^{49}$ Nous savons que des tentatives de résolution par radicaux de l'équation cubique ont été réalisées par des savants arabes dès le XII ${ }^{\mathrm{e}}$ siècle; ce qui conduit, bien entendu, à s'interroger sur les sources possibles du savant pisan du XIV ${ }^{\mathrm{e}}$ siècle.

Les trois manuscrits italiens analysés par Van Egmond n'indiquent ni auteur, ni date de traduction. L'identité de l'auteur et la date de composition du traité n'étaient alors connues que par leur mention dans la version hébraïque due à Mordekhay Finzi, lequel indique de surcroît l'auteur et la date de la copie qui avait servi de base à sa traduction: un certain Yacomo di Yerushali, demeurant à Mantoue, en 1429. Finzi ajoute qu'il a commencé cette traduction "à partir de la langue des chrétiens en hébreu" le mercredi 24 novembre 1473.50

48 MSS Oxford, fol. 69a; Paris, fol. 299b. La comparaison de l'argument avec celui qui figure dans le texte arabe (et latin) montre que l'argumentation y est analogue, mais la formulation, l'ordre des conclusions et le lettrage des figures diffèrent. Finzi a-t-il lui-même pallié la lacune? A-t-il consulté une autre source?

${ }^{49}$ W. Van Egmond, "The Algebra of Master Dardi of Pisa", Historia Mathematica, 10 (1983): 399-421.

${ }^{50}$ Sur la base du manuscrit Paris, BnF, MS héb. 1029, fols. 194b-234a, Van Egmond indiquait la date de 1469 pour la copie de Yacomo. Nous avons signalé, pour notre part, qu'il ne convenait pas de lire 1469 (fol. 196a) mais bien 1429, date figurant dans un autre manuscrit parisien, Paris, BnF, MS héb. 1033, fols. 
Deux autres résultats sont venus préciser la nature du texte original italien et de la version hébraïque. Nous connaissons maintenant une copie autographe de Finzi, ${ }^{51}$ et une autre copie du texte italien, appartenant initialement à une collection privée et déposée désormais à la bibliothèque de l'université de Tempe (Arizona). ${ }^{52}$ Qui plus est: ce codex, analysé par B. Hughes, comporte 35 gloses en hébreu (représentant quelque 800 mots). À la question posée par Hughes en conclusion de son article, ${ }^{53}$ nous sommes en mesure de répondre: oui, ces gloses sont bien de la main de Finzi; le manuscrit d'Arizona est effectivement le modèle utilisé par Finzi pour sa traduction en hébreu. ${ }^{54}$ Précisons que la traduction de Finzi s'interrompt avec la résolution de l'équation $\mathrm{n}^{\circ} 51$. Nous pouvons toutefois affirmer qu'il avait sans doute "préparé" la totalité de sa traduction: en témoigne une glose dans le manuscrit italien, qui n'est autre que l'énoncé (en hébreu) d'une des quatre équations irréductibles de degré strictement supérieur à 2. Ce passage intervient, dans l'original italien, après la résolution des 194 autres types d'équation. ${ }^{55}$ Du reste, la présentation de

91a-150b; voir notre "La littérature mathématique hébraïque en Europe ( du XI au XVI ${ }^{\text {e }}$ siècle)", dans C. Goldstein, J. Gray, J. Ritter (éds), L'Europe mathématique. Mythes, histoires, identités (Paris, 1996), pp. 84-99, voir n. 29.

${ }_{51}$ Jérusalem, National and University Library of the Hebrew University, MS $8^{\circ}$ 3915, fols. 1a-43a. Curieusement, ce codex a laissé échapper (?) les premiers feuillets de la copie de Finzi (correspondant à Paris 1029, fols. 194b-196a et à Paris 1033, fols. 91a-93a). Les deux manuscrits parisiens n'ont donc pas été copiés sur le texte autographe de Finzi, tel que nous le connaissons.

${ }^{52}$ B. Hughes, “An early 15th-century algebra codex: A description”, Historia Mathematica, 14 (1987): 167-72.

53 "Did Finzi use the Arizona State copy of Dardi's text as the exemplar of his own translation into Hebrew?", ibid., p. 172.

${ }^{54}$ On ne peut évidemment pas exclure que Finzi ait disposé d'une autre copie de la source. J'ai pu consulter le manuscrit de Tempe grâce à la courtoisie de M. Folkerts (Munich) qui m'en a communiqué un microfilm; qu'il en soit remercié. Dans l'analyse paléographique qui m'a permis d'identifier l'écriture de Finzi dans les gloses du manuscrit, j'ai été aidé par M. Garel (BnF), dont les compétences ont contribué à lever les doutes que je pouvais avoir; je l'en remercie vivement. De fait, nous disposons d'une preuve supplémentaire de l'identité du glossateur; en effet, dans sa version hébraïque, Finzi achève la présentation des 194 types d'équations résolubles de "façon générale" par les mots suivants: "les chapitres mentionnés ci-dessus sont traités par des règles générales, comme il apparaît dans la suite du cahier [jusque] au feuillet 121" (Paris 1029, fol. 195b); ce sont bien les mots qui figurent en hébreu dans le texte italien (MS Arizona, fol. 7b), à l'exception de l'indication du feuillet; toutefois, dans la marge gauche figure, en lettres hébraïques, la mention numérique 121; or c'est bien au folio $121 \mathrm{du}$ manuscrit d'Arizona que s'achève la résolution de ces types d'équations.

55 MS Arizona, fol. 121a. 
ces quatre types d'équation (et non leur résolution) figure bien dans le texte hébraïque qui nous est parvenu, puisqu'elle intervient à la suite de la présentation des 194 autres:

Après cela, je poursuivrai avec quelques chapitres ( = types d'équations), lesquels sont résolus par des règles non générales; toutefois ces règles sont bien valides et applicables dans des conditions particulières aux chapitres en question. Ce sont ceux que j'écris:

chapitre 1: une chose, un bien et un cube égal à un nombre

chapitre 2: une chose, un bien, un cube, un bien de bien (censo di censo) égal à un nombre

chapitre 3: une chose, un bien et un bien de bien égal à un nombre et un cube

chapitre 4: une chose et un bien de bien égal à un nombre, un bien et un cube $[\ldots] .{ }^{56}$

Finzi avait-il pris la mesure de l'importance de la présence de ces équations de degré supérieur à 2 ? Nous ne pouvons rien en dire.

La recherche n'a pas encore permis de tracer par quelles voies, quand il y en eut, les résultats établis par les savants arabes dans ce domaine ont pu circuler en Europe avant le XIV siècle. Toutefois des parallèles sont d'ores et déjà possibles. C'est ainsi que R. Rashed a signalé les tentatives d'al-Sulamī, au $\mathrm{XII}^{\mathrm{e}}$ siècle, pour résoudre par radicaux l'équation cubique $x^{3}+a x^{2}+b x=c$, qui n'est autre que l'équation $\mathrm{n}^{\mathrm{o}} 1$ ci-dessus. En imposant la condition $a^{2}=3 b$, al-Sulamī donne une solution réelle positive: $x=R_{3}\left(a^{3} / 27+\mathrm{c}\right)-a / 3$ où $\mathrm{R}_{3}$ désigne la racine cubique; et nous savons bien que la condition imposée permet de ramener l'équation proposée à une cubique pure, et donc sa résolution à l'extraction d'une racine cubique. ${ }^{57}$

Pour évaluer l'intérêt d'un tel rapprochement, indiquons la démarche de Maestro Dardi. Ayant indiqué précédemment que sa formule de résolution ne vaut que pour certains cas particuliers qui l'illustreront, il propose bien une procédure générale applicable à un sous-ensemble d'équations cubiques:

Quand les choses et les biens et les cubes sont égaux au nombre, tu dois diviser toute l'équation par la quantité des cubes; ensuite tu divises les choses par les biens; ce qui en résulte, prends-en le cube; ajoute ce produit au nombre; de la racine cubique de cette somme retranche le quotient des choses par les biens: c'est ce que vaut la chose. ${ }^{58}$

56 MS Paris 1029, fol. 195b.

${ }^{57}$ Rashed, "L'algèbre”, pp. 40-1.

${ }^{58}$ L'équation cubique ne figure pas dans MS Arizona (annoté et traduit par Finzi), lequel est différent, par bien des aspects, des trois manuscrits localisés en 
Traduisons ces instructions dans notre écriture symbolique. Une fois normalisée (on a divisé par le coefficient des termes de degré 3 ), notre équation devient $x^{3}+a x^{2}+b x=c$. La démarche suivie permet de calculer successivement: $b / a$, $(b / a)^{3},(b / a)^{3}+c, R_{3}\left[(b / a)^{3}+c\right], x=R_{3}\left[(b / a)^{3}+c\right]-b / a$. Sachant que la condition de validité de cette formule est $a^{2}=3 b$, soit $b / a=a / 3$, on retrouve bien la formule de résolution signalée par al-Sulamī. Et la condition, non énoncée par Dardi, est bien satisfaite dans l'exemple numérique qui suit:

Un homme a prêté 100 livres à un autre; au bout de trois années, il en reçoit 150 livres, capital et intérêt annuel. Je demande: à combien la livre a-t-elle été prêtée par mois?59

L'auteur procède comme suit. Il choisit comme inconnue $(C)$ le taux d'intérêt mensuel, exprimé en deniers (denari). Au bout d'un an, la livre produit un intérêt de $12 C$, exprimé en deniers, soit $1 / 20 C$, exprimé en livres ( 1 livre $=20$ sous, 1 sou $=12$ deniers). 100 livres produisent donc au bout de la première année un intérêt d'un montant, en livres, de 100/20C, soit $5 C$; le nouveau capital est $100+5 C$. Au bout de la deuxième année, le montant de l'intérêt est $5 C+(5 C) \times 1 / 20 C$, soit $5 C+1 / 4 C^{2}$; le nouveau capital est $100+10 C+1 / 4 C^{2}$. Au bout de la troisième année, le montant de l'intérêt est $5 C+10 / 20 C^{2}+(1 / 4) \times$ $(1 / 20) C^{3}$, soit $5 C+1 / 2 C^{2}+1 / 80 C^{3}$; et le nouveau capital est donc $100+15 C+3 / 4 C^{2}+1 / 80 C^{3}$; d'où l'équation $100+15 C+$ $3 / 4 C^{2}+1 / 80 C^{3}=150$. Après transposition et réduction, il vient $C^{3}+60 C^{2}+1200 C=4000$.

La solution proposée par l'auteur est $C=R_{3}\left[(1200 / 60)^{3}+\right.$ 4000]-1200/60; $R_{3}$ désignant la racine cubique. La livre rapporte donc au prêteur près de 3 deniers par mois.

Aucune justification n'est apportée, mais on aura remarqué que les coefficients numériques (donc les données du problème) sont tels que le carré du coefficient du terme de degré 2 est égal au triple du coefficient du terme de degré 1; c'est la condition générale, nous le savons, pour réduire l'équation à une cubique

Italie et analysés par Van Egmond (qui prépare l'édition critique de l'original italien). En attendant cette édition critique, on peut consulter la transcription du manuscrit de Sienne, précédée d'une brève introduction, dans: Maestro Dardi (sec. XIV). Alibraa Argibra, dal manoscritto I. VII. 17 della Biblioteca Comunale di Siena, éd. R. Franci, Quaderni del Centro Studi della Matematica Medioevale $\mathrm{n}^{\circ}$ 26 (Sienne, 2001); voir p. 270.

59 Ibid.: Uno presta a un altro lire 100, e in capo di 3 anni egli riceve lire 150 tra chapitale et merito a fare chapo d'anno. Adimando a quanto fu prestata la lira lo mese. 
pure, dont la résolution se ramène à une extraction de racine cubique.

Quelles sont les traces repérables en hébreu d'une nouvelle activité algébrique - quelque modeste qu'elle fût -, issue des textes que nous venons de présenter. Sur ce point, les éléments de réponse sont aussi fragmentaires que la tradition textuelle en question. Postérieurement à Finzi (en termes de chronologie et non de généalogie), nous connaissons des textes d'arithmétique ou de géométrie, compilés, traduits ou adaptés de sources italiennes, et comportant des développements algébriques. ${ }^{60}$ Ces textes demandent à être analysés.

\section{REMARQUES DE CONCLUSION}

Comment caractériser les textes que nous avons rassemblés? et plus généralement les efforts dont ils témoignent pour faire connaître en hébreu la science algébrique en marche? Nous l'avons déjà souligné: les contours de la littérature mathématique hébraïque sont encore mal définis; c'est encore plus vrai pour ces fragments d'algèbre dont certains étaient ignorés il y a peu. On peut toutefois formuler quelques remarques avant de proposer de nouvelles pistes d'investigation.

Les plus anciens recours aux règles de l'algèbre sont localisables dans des textes ou des passages de géométrie pratique; la légitimité de ce recours est celle qui est à l'œuvre dans les livres arabes du même genre: équations quadratiques, équations ou systèmes d'équations linéaires. Les six équations canoniques classiques, généralement non référées au nom du père-fondateur de la science de l'algèbre, suffisent largement à expliciter les règles de mensuration de surfaces et de volumes.

Nous avons vu qu'Abraham bar Hiyya, et son cadet Abraham ibn Ezra, au XII ${ }^{\mathrm{e}}$ siècle, recouraient à une algèbre que nous avons qualifiée de "muette", au sens où la terminologie technique de l'algèbre et le mot même d'algèbre en étaient

\footnotetext{
${ }^{60}$ Parmi les textes dont nous avons juste relevé l'intérêt signalons: Gad Astruc ben Jacob (actif au début du $\mathrm{XVI}^{\mathrm{e}}$ siècle), connu comme copiste de textes mathématiques, est l'auteur d'une arithmétique "classique", suivie de 452 problèmes de tous genres, dont certains sont résolus par les "posicioni dil alzibra", voir Londres, British Library, MS Add. 27 039, fols. 3a-116a. Une traduction (adaptation?) anonyme de la Summa de Luca Paccioli: 387 problèmes répartis en trois chapitres et présentés comme des "extraits du livre de Fra Luqa"; ibid., fols. 116a-184b.
} 
absents. ${ }^{61}$ Pour autant, la présentation des six équations canoniques est bien attestée en hébreu, en dehors des textes proprement algébriques. À ce jour nous en connaissons trois exemples, et il ne serait pas étonnant qu'on en découvre d'autres. ${ }^{62}$

Le livre d'al-Hașșār, traduit en hébreu au XIII ${ }^{\mathrm{e}}$ siècle, ressortit à un autre genre, très répandu dans la littérature arabe: un manuel d'arithmétique se référant à l'algèbre comme à "une" technique de recherche de nombres inconnus à partir de nombres connus donnés, les autres techniques citées étant généralement la règle de trois ou les règles de fausse position.

L'épître du nombre d'Isaac ben Salomon, à la fin du XIV siècle, est un cas plus intriguant. On l'a montré: il s'agit de la traduction amplement commentée du célèbre petit ouvrage d'Ibn al-Bannā', Résumé des opérations du calcul; l'algèbre qui y est développée n'est pas d'un niveau élémentaire; elle est proposée comme une composante d'une théorie générale de l'arithmétique des expressions algébriques. Nous sommes là dans la tradition algébrique d'al-Karajī. Isaac ben Salomon, en Castille, est un astronome de renom, un arabisant de talent; il nous dit s'être rendu en pays d'Islam (Grenade? Afrique du Nord?), y avoir acquis l'ouvrage arabe et quelques autres de même nature, s'y être instruit auprès de maîtres de la science du nombre, avant de l'enseigner à son tour quelques années plus tard en Sicile. Nous connaissons d'autres savants juifs castillans, contemporains ou maîtres d'Isaac, ayant réuni sans doute les mêmes compétences; il est difficile d'admettre que les

${ }^{61}$ Le texte d'Ibn Ezra a été récemment édité, traduit et commenté; voir T. Lévy et C. Burnett, "Sefer ha-Middot: A mid-twelfth-century text on arithmetic and geometry attributed to Abraham Ibn Ezra", Aleph 6 (2006): 57-238.

${ }^{62}$ Nous avons étudié l'un d'entre eux dans T. Lévy, "A newly discovered partial Hebrew translation of al-Khwārizmī's Algebra", Aleph, 2 (2002): 225-34. Les deux autres exemples appellent une analyse que nous pensons publier prochainement: Paris, BnF, MS hébr. 1081, fols. 62b-67a et Mantoue, Biblioteca comunale, MS Ebr. 17, fols. 128b-130b. Dans le deuxième cas, le codex est celui-là même qui reproduit l'Algèbre d'Abū Kāmil et le copiste en est Mordekhay Finzi lui-même. L'ouvrage qui contient l'exposé des six équations "khwarizmiennes", avec démonstrations géométriques, est traduit (ou adapté) d'un ambitieux ouvrage italien en 11 parties (les titres en sont indiqués en introduction), dont seules les cinq premières nous sont parvenues en hébreu (MS Mantoue 17, fols. 86b-111b, 124a-152a). Ni l'auteur, ni le traducteur en hébreu ne sont indiqués. Euclide, Campanus et Jordanus y sont cités. L'auteur de cette compilation indique en introduction qu'il a exploité plusieurs ouvrages et qu'il offre à son lecteur géomètre les moyens d'effectuer la "mensuration (medida)" de toutes les figures géométriques, planes ou solides. 
initiatives d'Isaac ont relevé de sa seule curiosité individuelle, et non d'un "climat" d'échanges intellectuels et d'intérêt nourri par des ouvrages.

Avec l'Italie du $\mathrm{XV}^{\mathrm{e}}$ siècle, on semblait devoir s'éloigner de l'Espagne arabe. Et pourtant, à considérer le travail de Finzi sur Abū Kāmil (la copie révisée du grand livre d'algèbre, la rédaction d'un exposé sur "les six problèmes"), nous voilà ramenés à l'Espagne et aux ressources qu'elle recèle encore pour la recherche. Si notre conjecture relative à la traduction en hébreu du grand livre d'Abū Kāmil est valide - réalisée par un savant juif hispanophone, destinée à un public aussi hispanophone -, tout porte à croire qu'elle vit donc le jour en Espagne, au plus tard au milieu du $\mathrm{XV}^{\mathrm{e}}$ siècle. 